\title{
Elastic Neural Net for the Earthquake Epicenter Search
}

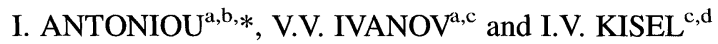 \\ ${ }^{\mathrm{a}}$ International Solvay Institutes for Physics and Chemistry, CP-231, ULB, Bd. du Triomphe, 1050 Brussels, Belgium; ${ }^{\mathrm{b}}$ Theoretische Natuurkunde, Free \\ University of Brussels, Brussels, Belgium; ' Joint Institute for Nuclear Research, Dubna 141980, Russia; ${ }^{\mathrm{d}}$ Max-Planck-Institut für Physik München, \\ Werner Heisenberg Institut, Föringer Ring 6, D-80805, Munich, Germany
}

(Received 20 March 2001)

\begin{abstract}
Automatic processing of seismic data is today a key element in the efforts to achieve high quality seismic systems. Automated procedures for locating seismic events with a network including arrays and single element seismometers usually incorporate back-azimuth estimates, arrival-time data, and associated uncertainties into a least-squares-inverse location algorithm. Such an algorithm is quite cumbersome and requires expanding a set of non-linear equations in a Taylor series. Second-order terms usually not included in the algorithm can be important if the initial estimate is far from the solution.

We propose to use elastic neural nets (ENN) to find the initial estimation in automated procedures of locating seismic events and discuss the results for simulated seismic events. The advantages of ENN are the simplicity of the algorithm, the fast convergence and the high efficiency.
\end{abstract}

Keywords: International data centers; Elastic net; Seismic event; Epicenter

\section{INTRODUCTION}

With the development of digital seismograph recording systems, the automatic processing of seismic data has become increasingly important. In particular, the everdecreasing cost and the increasing capacity of modern computers have made possible the implementation of very sophisticated automatic processing algorithms. Today, the limitations do not regard computer capacity, but rather shortcomings of the available automatic schemes.

In March 1978, the group of seismic experts established by the Conference of the Committee on Disarmament (CCD, 1978) of the United Nations submitted a report with recommendations regarding an international cooperative system to detect and identify seismic events. Such a system would become a key part of the verification of a comprehensive test ban treaty. The ad hoc expert group established by the CCD in 1976 has proposed the structure of the global system (Fig. 1). This international co-operative effort involves three main elements:

1. A systematic improvement in the observations reported from more than 50 seismological observatories around the globe.
2. An international exchange of these data over the Global Telecommunications System (GTS) of the World Meteorological Organization.

3. Processing of data at special International Data Centers (IDC) for the use of the participant states.

The ad hoc group considered that special IDC should be established for the global network. In order to achieve reliability acceptable to all, it was proposed that more than one international center have to be established, equipped with compatible hardware and software.

The main tasks of the international centers should be:

1. To receive data from the world network of seismic stations via the authorized Government facility of each State.

2. To apply agreed analysis procedures to available data for the estimation of the origin time, location, magnitude and depth of seismic events.

3. To associate the reported identification parameters with these events.

*Corresponding author. Address: International Solvay Institutes for Physics and Chemistry, CP-231, ULB, Bd. du Triomphe, 1050 Brussels, Belgium. E-mail: iantonio@pop.ulb.ac.be 


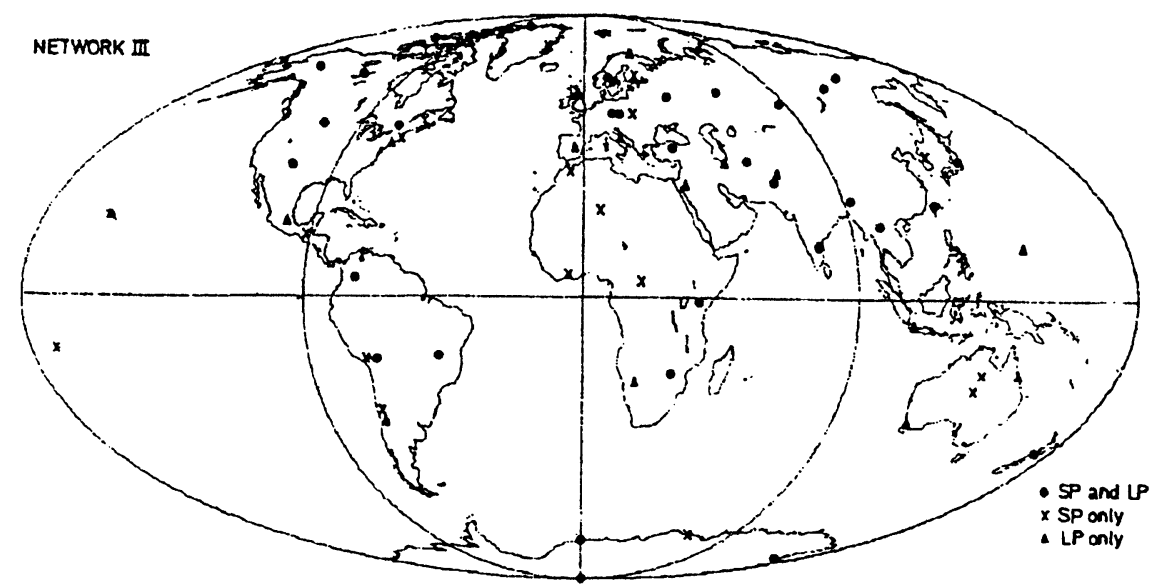

FIGURE 1 A possible global network of existing and planned seismic observatories proposed by the CD ad hoc group of seismic experts.

4. To distribute, in accordance with defined procedures and without interpretation of identification parameters, compilations of the complete results of these analyses.

5. To act as an archive for reported data and results of analysis on those data.

\section{SEISMIC EVENT LOCALIZATION PROCEDURE}

The determination of the seismic event location is one of the most important functions of the IDC. The results of the localization process, such as, the epicenter location, depth, the origin time, error estimates, form the primary data product of the IDC: the bulletin.

The procedure for event location calculates epicenters, confidence bounds, residuals and data importance using arrival times, back-azimuths, and slownesses from seismic phases recorded by stations at local, regional and teleseismic distances. Travel time, slowness, and azimuth data are, thus, incorporated simultaneously to solve the iterative non-linear least-squares problem for hypocentral locations.

The location procedure follows the approach of Jordan and Sverdrup (1981) as extended by Bratt and Bache (1988) to include azimuth and slowness data. The key feature of this procedure is that it allows a priori knowledge of the data variances to be incorporated along with the resultant a posteriori data residuals. This makes calculation of the error ellipses particularly robust.

In order to approximate better the event location in a truly heterogeneous media, while also maintaining the simplicity of travel time tables based on a onedimensional velocity model, various source-specific corrections can be made. These corrections usually take the form of travel time corrections, but azimuth and slowness corrections are also possible.

Let us present the basis of the location algorithm. Geiger's (1910) iterative, least-squares method and its various extensions and modern improvements (e.g. Flinn, 1965; Buland, 1976) are the basis of most numerical algorithms for earthquake location. A point seismic source is specified by a space-time vector $x$ of dimension $M \leq 4$. The inequality refers to the case when certain components, such as depth, are fixed throughout the calculation. This location vector is to be constrained by an $N$-component vector of the observed arrival times $t^{0}$. Formally, $x \in E^{M}$ and $t^{0} \in E^{N}$, where $E^{N}$ is the Euclidean space of dimension $N$. Since the number of observation points $N$ is usually large compared to $M$, the problem is overdetermined, although it may still be ill-posed. From the initial estimate $x_{0}$, an arrival-time vector $\bar{t}\left(x_{0}\right)$ is calculated by tracing rays through an average radial earth structure; the overbar indicates the assumption of spherical symmetry. By expanding $\bar{t}$ about $x_{0}$ we can write

$$
\overline{\Delta t} \equiv t(x)-t\left(x_{0}\right)=\mathbf{A} \cdot \Delta x+O\left[|\Delta x|^{2}\right],
$$

where $\Delta x \equiv x-x_{0}$ and $\mathbf{A}$ is an $N \times M$ matrix of partial derivatives evaluated at $x_{0}$. If we assumes that the rank $[\mathbf{A}]=M$, then a refined estimate of $x$ can be uniquely obtained from the residual vector $\overline{\Delta t} \equiv t(x)-t\left(x_{0}\right)$ by minimizing the norm of the error vector:

$$
e^{0}=\Delta t^{0}-\mathbf{A} \cdot \Delta x
$$

To account for the curvature of the $\bar{t}(x)$ manifold, the estimate of $\Delta x$ is added to $x_{0}$ and the procedure is iterated. The convergence is usually rapid in the teleseismic case.

In certain situations, especially for epicenters determined by sparse local networks and/or when locating local and near-regional events that lie outside the network, the non-linear aspects of the problem may be important (Flinn, 1965; Buland, 1976; Spence, 1980). Techniques, including non-linear contributions to local uncertainty and the shape of these non-linear confidence bounds, may be quite complex compared to the ellipsoids resulting from the linear approximation.

It becomes obvious that the calculation of the initial estimation of $x_{0}$ is the crucial point of the full location algorithm. We shall use an elastic neural net for estimating $x_{0}$. 

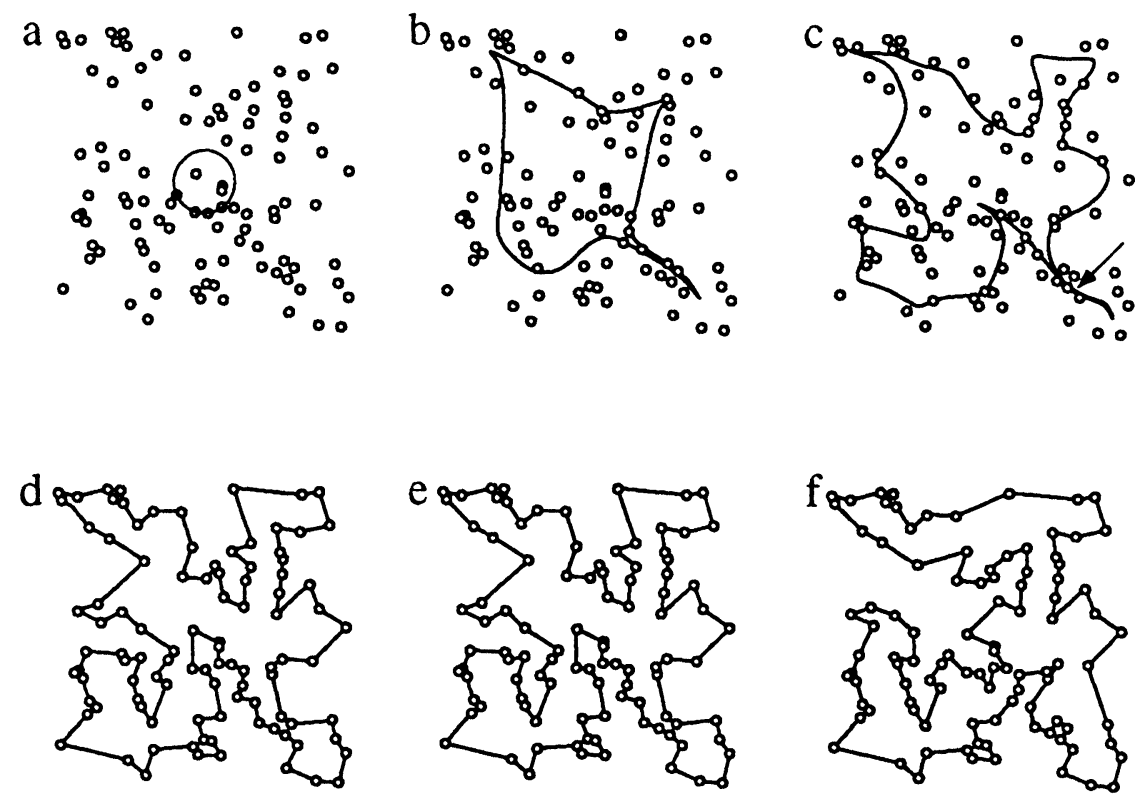

FIGURE 2 Example of the progress of the elastic net method for 100 cities.

\section{ELASTIC NET METHOD}

The elastic net is a kind of artificial neural net (Kisel et al., 1993) which has been used for event recognition in high energy physics.

The elastic neural net method can be illustrated on a simple example of the traveling salesman problem. The problem is:

Given the positions of $N$ cities, what is the shortest closed tour in which each city can be visited once?

One of the most successful approaches to the traveling salesman problem is the elastic neural net of Durbin and Willshaw (1987), $\dagger$ which can be understood as a number of beads connected by an elastic string to form a ring. The essence of the method is:

Using an iterative procedure, a circular closed path is gradually elongated non-uniformly until it eventually passes sufficiently near to all the cities to define a tour.

Let us denote the cities by $\vec{x}_{i}$. We are going to match these cities with template coordinates $\vec{y}_{a}$ such that the sum $\sum_{a}\left|\vec{y}_{a}-\vec{y}_{a+1}\right|$ is minimized and that each $\vec{x}_{i}$ is matched by at least one $\vec{y}_{a}$. Define $s_{i a}$ to be 1 if $a$ is matched to $i$ and 0 otherwise. The following energy expression (Peterson and Rognvaldsson, 1992) then minimizes a valid tour

$$
E\left(s_{i a}, \vec{y}_{a}\right)=\sum_{i a} s_{i a} \cdot\left|\vec{x}_{i}-\vec{y}_{a}\right|^{2}+\gamma \cdot \sum_{a}\left|\vec{y}_{a}-\vec{y}_{a+1}\right|^{2} .
$$

The coefficient $\gamma$ governs the relative strength between matching and tour length. Then the dynamical equations can be written as

$$
\begin{aligned}
\Delta \vec{y}_{a} & =\Delta \vec{y}_{a}^{(1)}+\Delta \vec{y}_{a}^{(2)} \\
& =\eta\left[2 \sum_{i} v_{i a} \cdot\left(\vec{x}_{i}-\vec{y}_{a}\right)+\gamma \cdot\left(\vec{y}_{a+1}-2 \vec{y}_{a}+\vec{y}_{a-1}\right)\right],
\end{aligned}
$$

where the decision value $v_{i a}$ of $a$ to be matched to $i$ is given by

$$
v_{i a}=\frac{\mathrm{e}^{-\left|\vec{x}_{i}-\vec{y}_{a}\right|^{2} / T}}{\sum_{b} \mathrm{e}^{-\left|\vec{x}_{i}-\vec{y}_{b}\right|^{2} / T}} .
$$

The "temperature" $T$ is decreasing during evolution.

The algorithm is a procedure for the successive recalculation of the positions of a number of points in the plane in which the cities lie. The points describe a closed path which is initially a small circle centered on the center of the distribution of cities and is gradually elongated non-uniformly to eventually pass near all the cities and thus define a tour around them (for details see the original paper Durbin and Willshaw, 1987). Each point on the path moves under the influence of two types of force (see Eq. (4)):

1. the first pulls towards the nearest cities;

2. the second pulls towards neighboring points on the path, acting to minimize the total path length.

By this process, each city becomes associated with a particular section on the path. The tightness of the association is determined by how the force contributed 


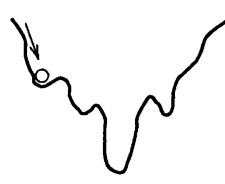

a)

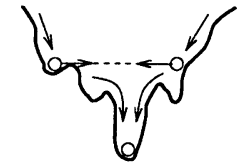

b)
FIGURE 3 (a) Global minimum search by a standard method and (b) the elastic net method.

from a city depends on its distance, and the nature of this dependence changes as the algorithm progresses. Initially all cities have roughly equal influence on each point of the path. Subsequently, a larger distance becomes less favored and each city gradually becomes more influenced for the points on the path closest to it.

Usually, the ENN finds a solution close to the best one, that is practically enough in many applications. Thus, in our case we should find a solution, which could be used for making a decision about a vertex existence and for using it as an initial approximation for the least squares method.

An example of the TSP solution for 100 cities, using ENN is presented in Fig. 2.

\section{ELASTIC NET FOR THE EARTHQUAKE PROBLEM}

Let us formulate the problem of searching for an initial estimation of a seismic event epicenter. Data from a network of arrays and single element seismometers include arrival times, back-azimuths, and slownesses from seismic phases recorded by stations at local, regional and teleseismic distances. This information could be represented by the distance and the direction towards the earthquake center registered by seismometers with corresponding errors that give confidence ellipsoids for each station. Parameters and location of an ellipsoid depend on the technical characteristics of the station and the distance to the event source.

For testing the elastic net algorithm, we simulate data from six "correct" stations and three "noise" stations (see Fig. 4). This usually happens analyzing seismic data at IDC. A "correct" radius (detection error) is random up to 5.0 relative units. A "correct" center location is generated with a random shift within the generated detection error with respect to the generated event location and with random angle distribution. "Noise" detection error is the same as for "correct" stations but locations are shifted randomly in addition up to 5.0 relative units to be far from the true location. $\neq$

Let us

Define an epicenter of a seismic event as the geometrical point with a maximum density of confidence ellipsoids.

The earthquake location procedure at presence of noise data can be considered as a problem of minimization of a

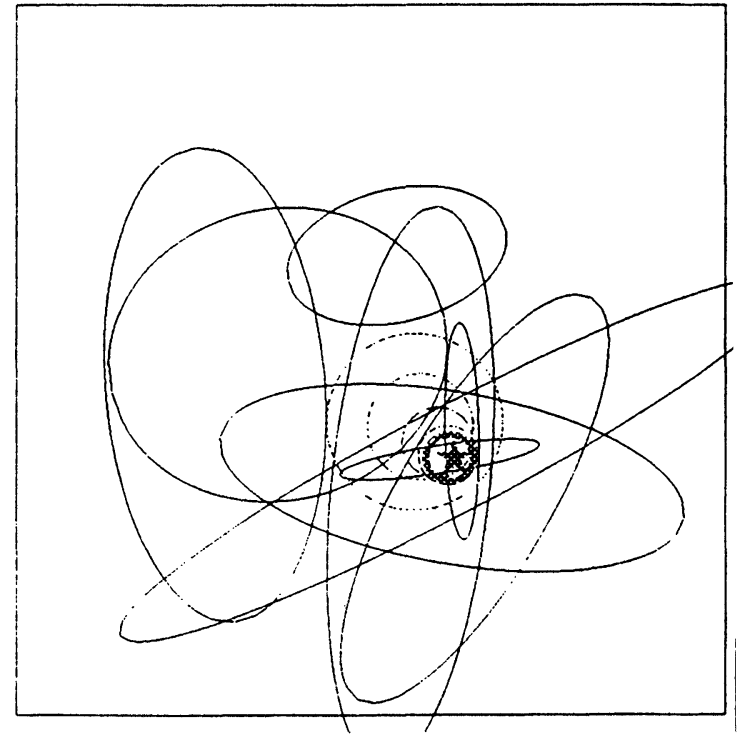

FIGURE 4 An evolution of the elastic net on a data from six correct and three noise seismic stations.

function of many variables with many local minima. All known standard methods of minimization (Press et al., 1986) start with a point on the function surface and minimize the function in a local area around it, at each step of the iteration procedure. In this way, there is a significantly non-zero probability to stop at a local minimum. It is possible to build methods against local minima to save the standard methods, but to do so the complexity of our program increases significantly. The only reliable way is to start with a good initial approximation, which cannot be easily found in our case. So the use of the standard minimization methods is not suitable.

Contrary to the standard methods, we propose to use an elastic ring surrounding the global minimum and covering the region where the parameters are likely to be found. This ring will be squeezed, catching on all local minima until the global minimum will be reached (Kisel and Kovalenko, 1996). Graphically, this method is represented in Fig. 3.

We construct the algorithm on the basis of an elastic ring by introducing two types of force:

1. Attraction of the ring to all ellipsoids placing it at the condensation area of the ellipsoids. Here confidence ellipsoids play a role of cities in the traveling salesman problem. The attraction force of the ring to an ellipsoid depends on its distance and location accuracy given by a station. The sensitivity region of the ring is decreasing during iterations being more confident at the end of the process.

2. Squeezing of the ring leading to localization of the source region. The influence of this force increases with algorithm iterations. The radius of the ring corresponds to the most probable area surrounding the

\footnotetext{
${ }^{\ddagger}$ A similar problem appears when searching for the reaction vertex in high energy physics experiments (Kisel et al., 1997).
} 


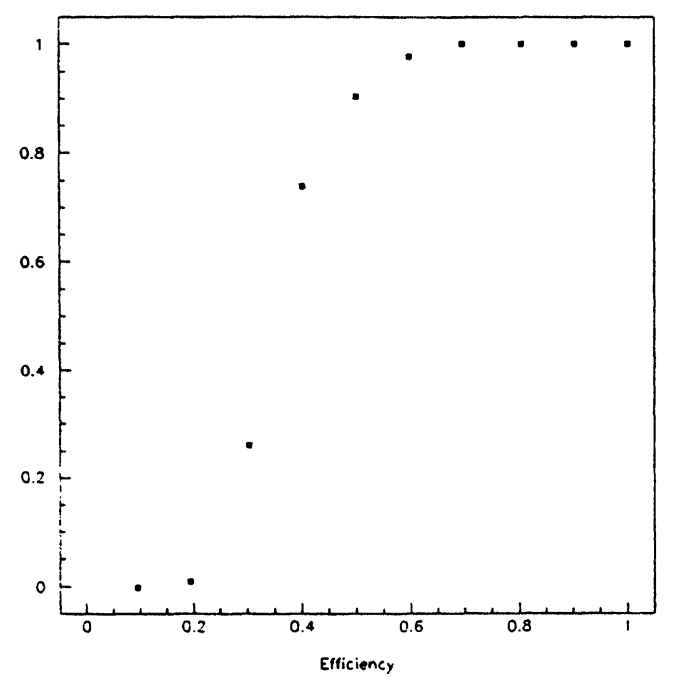

FIGURE 5 Efficiency of the epicenter searching depending on seismic data efficiency.

epicenter.

\section{RESULTS AND CONCLUSION}

Figure 4 shows the result of the application of the ENN to the earthquake location searching, using data from nine seismic stations. Here the star denotes the epicenter of the simulated earthquake and six solid circles are simulated confidence circles given by seismic stations. Other stations give noisy data and one can see three circles far from the epicenter. Dashed circles demonstrate the iterative process of the elastic net that converges near the epicenter. This found position can be used as the initial estimation in the procedure of the epicenter calculation (Eq. (2)).

The advantages of the method are the simplicity of the algorithm, the fast convergence and the high and stable reconstruction efficiency. One can see on Fig. 5 that the algorithm is able to find the epicenter even with $50 \%$ wrong seismic data.

\section{Acknowledgements}

We acknowledge the International Solvay Institutes for Physics and Chemistry and the Belgian Government through the Inter-university Attraction Poles for financial support. We are grateful to Prof. I. Prigogine for encouragement and support.

\section{References}

Bratt, S.R. and Bache, T.C. (1988) "Locating events with a sparse network of regional arrays", Bull. Seism. Soc. Am. 78, 780.

Buland, R. (1976) "The mechanics of locating earthquakes", Bull. Seism. Soc. Am. 66, 173.

CCD (1978) Conference of the Committee on Disarmament Paper CCD/558, 1978, Geneva.

Durbin, R. and Willshaw, D. (1987) "An analogue approach to the traveling salesman problem using an elastic net method", Nature 326, 689.

Flinn, E. (1965) "Confidence regions and error determinations for seismic event location", Rev. Geophys. 3, 157.

Geiger, L. (1910) "Herdbestimmung bei Erdbeben ans den Ankunftzeiten”, K. Gessell. Wiss. Goett. 4, 331.

Jordan, T.H. and Sverdrup, K.A. (1981) "Teleseismic location techniques and their application to earthquake clusters in the south-central Pacific", Bull. Seism. Soc. Am. 71, 1105.

Kisel, I. and Kovalenko, V. (1996) "Elastic net for broken multiple scattered tracks”, Comp. Phys. Commun. 98, 45.

Kisel, I., Neskoromnyi, V. and Ososkov, G. (1993) "Applications of neural networks in experimental physics", Phys. Part. Nucl. 24(6), 657.

Kisel, I., Konotopskaya, E. and Kovalenko, V. (1997) "Elastic neural net for track and vertex search", Fifth International Workshop on Software Engineering, Artificial Intelligence, Neural Nets, Genetic Algorithms, Symbolic Algebra and Automatic Calculation AIHENP'96, Lausanne, Switzerland, September 1-6, 1996; Nucl. Instr. Meth. A389, 167.

Peterson, C. and Rögnvaldsson, T. (1992) "Introduction to artificial neural networks", 1991 CERN School of Computing, Ystad, Sweden, August 23-September 2, 1991, CERN 92-02, p. 113.

Press, W.H., Flannery, B.P., Teukolsky, S.A. and Vetterling, W.T. (1986) Numerical Recipes: the Art of Scientific Computing (Cambridge University Press, Cambridge).

Spence, W. (1980) "Relative epicenter determination using P-wave arrival-time differences", Bull. Seism. Soc. Am. 70, 171. 


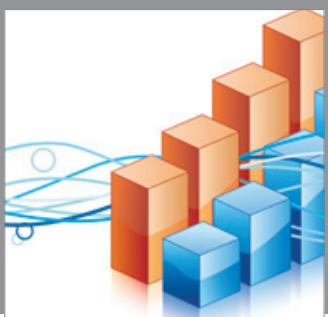

Advances in

Operations Research

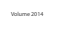

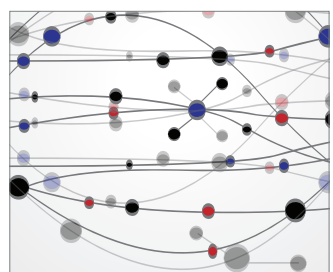

\section{The Scientific} World Journal
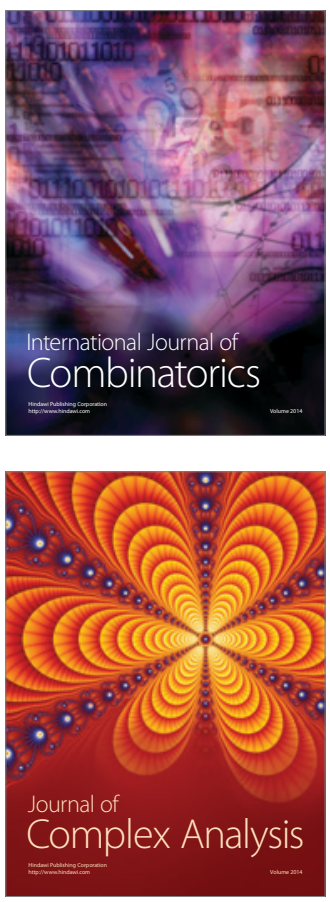

International Journal of

Mathematics and

Mathematical

Sciences
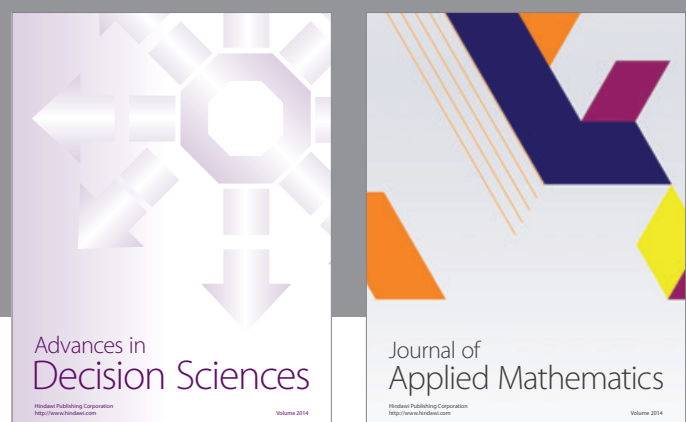

Journal of

Applied Mathematics
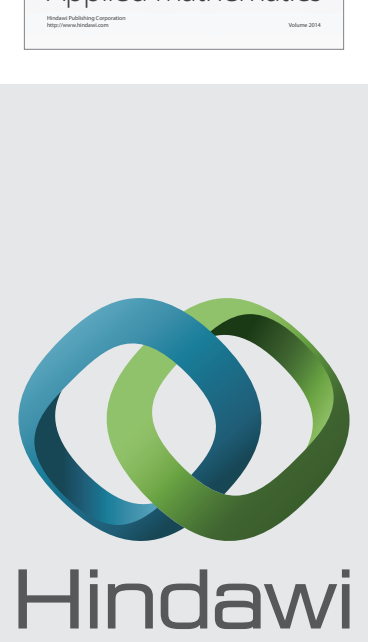

Submit your manuscripts at http://www.hindawi.com
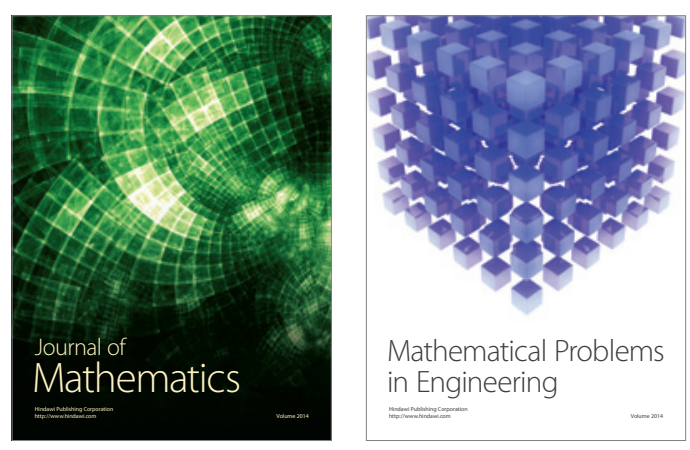

Mathematical Problems in Engineering
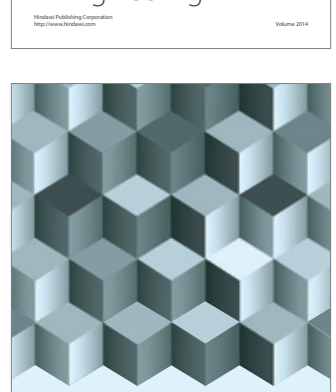

Journal of

Function Spaces
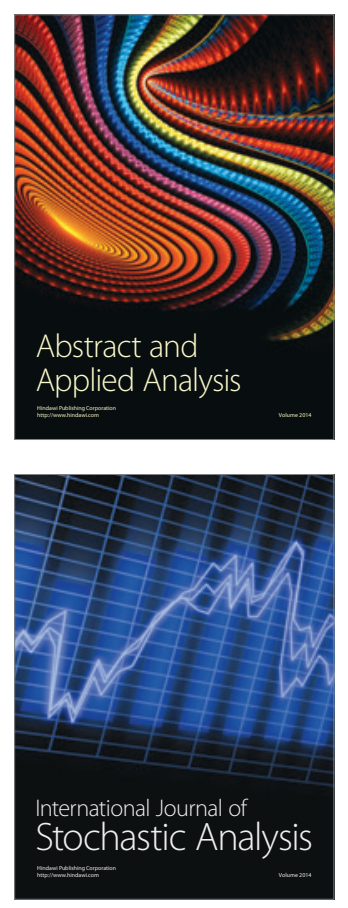

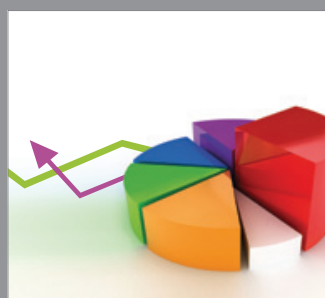

ournal of

Probability and Statistics

Promensencen
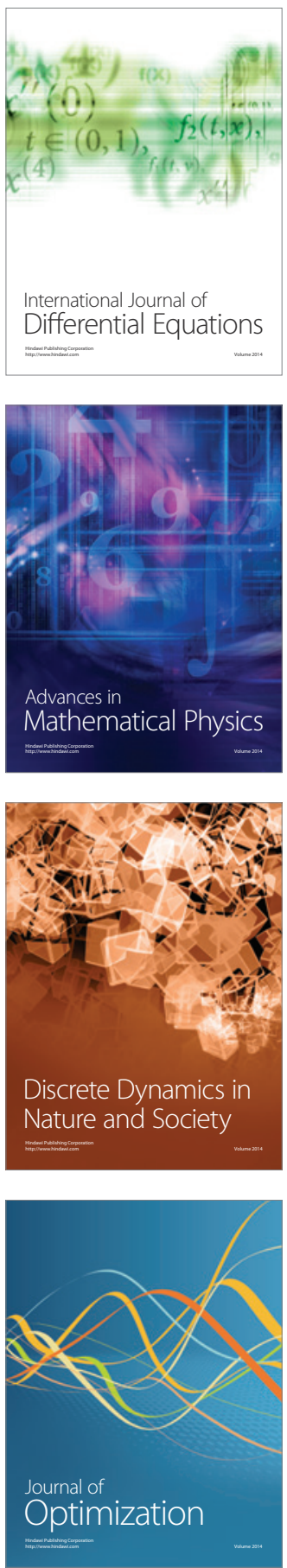